Herzschr Elektrophys 2014 - 25:57-58

DOI 10.1007/s00399-014-0317-7

Online publiziert: 6. Mai 2014

(c) Springer-Verlag Berlin Heidelberg 2014

\section{Thomas Deneke}

Klinik für Kardiologie II (Interventionelle Elektrophysiologie), Herz- und Gefäßklinik Bad Neustadt, Bad Neustadt a. d. Saale, Deutschland

\section{Shelter from the storm}

\section{Vorgehen bei Patienten mit elektrischem Sturm}

Seit den 1980er Jahren hat sich die Prävention des plötzlichen Arrhythmie-bedingten Herztods mit implantierbaren Defibrillatoren (ICDs) als Standard etabliert. Hierdurch ist ein neues Patientenkollektiv entstanden, welches sich durch gehäuftes Auftreten von ventrikulären Arrhythmien, vor allem monomorphen ventrikulären Tachykardien (VTs), definiert. Diese werden effektiv durch den ICD terminiert. Das Auftreten multipler ( $\geq 3$ ) distinkter Episoden von ventrikulären Arrhythmien innerhalb von 24 Stunden wird als elektrischer Sturm definiert. Während sich die klassische Definition auf Patienten mit ICDs beschränkte, sollten auch Patienten ohne eine bisherige ICD-Implantation eingeschlossen werden, da durch die optimierte frühzeitige prähospitale Versorgung bei akut bedrohlichen Herzrhythmusstörungen die Überlebenschancen dieser bisher ungeschützten Patienten höher ist und somit in der Folge die Frage der Prävention von Rezidiv-Ereignissen aufkommt.

Generell stellen Patienten mit gehäuften Episoden anhaltender ventrikulärer Arrhythmien ein Patientenkollektiv dar, das einer schnellen und effektiven Therapie bedarf. Außerhalb eines akuten Myokardinfarkts existieren multiple auslösende Faktoren für einen elektrischen Sturm, wie z. B. Medikamenteneinflüsse, Elektrolyt-Imbalance, aber auch kardiale Faktoren wie Aggravation einer Herzinsuffizienz. Eine schnellstmögliche Identifikation und adäquate Therapie scheinen die Grundsäulen der effektiven Stabilisierung von Patienten mit elektrischem Sturm zu sein.
Bisher existieren lediglich rudimentäre Daten zur Häufigkeit und Mortalität des elektrischen Sturms in der generellen Bevölkerung. Es kann allerdings davon ausgegangen werden, dass Patienten mit elektrischem Sturm diesen häufig akut nicht überleben, vor allem wohl auch, da strukturierte Therapiestrategien fehlen. In dem hier präsentierten Schwerpunktheft werden mögliche auslösende Faktoren, spezifische Charakterisierungen der Arrhythmien bei elektrischem Sturm und Handlungsstrategien diskutiert. Ziel ist es dabei, dem Leser Material an die Hand zu geben, um einen Therapie-Pathway zu entwickeln, der eine optimierte und angepasste Therapiekaskade für die Behandlung des elektrischen Sturms ermöglicht. Eine Fokussierung auf spezielle medikamentöse, sedierende und evtl. ausgeweitete Interventionen in der Therapie ermöglicht die Entwicklung eines dezidierten Eingriffsplans für die Notfalltherapie. Allerdings fehlen bisher Daten zum akuten und mittelfristigen Benefit eines standardisierten Vorgehens. Erstmals wird im Rahmen des Electrical-Storm-Netzes Bayern versucht, unterschiedliche Therapiestrategien insbesondere auch auf ihre zeitliche Sinnhaftigkeit und Effektivität zu vergleichen.

Initiales Ziel der Behandlung des elektrischen Sturms ist die akute Rhythmusstabilisierung, die häufig mit wenig Aufwand (antiarrhythmische Therapie, Sedierung und bei selektierten Patienten evtl. auch gezielte Koronartherapie) erzielt werden kann. Eine Ausweitung der Therapie bei Ineffizienz der initialen rhythmusstabilisierenden Maßnahmen sollte dann jedoch an kardiologischen Spezialzentren mit der Möglichkeit einer hämodynamischen Unterstützungstherapie und elektrophysiologischen Intervention erfolgen. Um hier ein Ineinandergreifen der Therapieoptionen $\mathrm{zu}$ ermöglichen, erscheint die Entwicklung von Netzwerken kooperierender Kliniken mit speziell dedizierten interventionellen ElektrophysiologieZentren angebracht. Aber auch in diesen sollte eine spezielle Fokussierung auf $\mathrm{Pa}$ tienten mit ventrikulären Arrhythmien erfolgen oder bereits vorhanden sein. Strukturierte Eingriffsprotokolle erscheinen hier ebenso notwendig, um möglichst effektiv und schnell intervenieren zu können. Es konnte gezeigt werden, dass gerade eine vorgehaltene Spezialunit zur optimierten Therapie dieser Patienten eine wesentlich wirksamere Therapie ermöglicht und weiteres Optimierungspotenzial aufdeckt. Vergleichsuntersuchungen zur Identifikation der besten Therapiestrategien können nur im Rahmen großer Registerdaten ermöglicht werden, da auch in ausgewiesenen Zentren große Fallzahlen fehlen.

Wie in den folgenden Artikeln beschrieben, sind die Anforderungen an ein Interventionszentrum im Rahmen des elektrischen Sturms vielfältig und insbesondere auch auf dem Gebiet der interventionellen Elektrophysiologie vielschichtig. Die Notwendigkeit, komplexe, individuell abgestimmte Ablationsprozeduren inklusive epikardialem Vorgehen (insbesondere bei Patienten mit nichtischämischen Kardiomyopathien) durchzuführen, erfordert eine hohe Expertise der elektrophysiologischen Interventionszentren als Partner in einem überregionalen Netzwerk kooperierender Kliniken. Auch 
wenn ein Großteil der invasiven elektrophysiologischen Prozeduren früh-elektiv - zur Modifikation des zugrunde liegenden Substrats und somit Verhinderung von Rezidiv-Ereignissen - erfolgen kann, existiert doch ein geringer Prozentsatz an Patienten, bei denen akut innerhalb der ersten Stunden nach Beginn eines elektrischen Sturms eine komplexe Ablationsprozedur zur Unterbrechung der lebensbedrohlichen Arrhythmien angeboten werden muss. Die Etablierung und Strukturierung von kooperierenden Klinik-Netzwerken stellt eine wesentliche Herausforderung der nächsten Jahre dar, um Patienten mit elektrischem Sturm optimal zu behandeln.

Die hier zusammengestellten Artikel sollen dem Leser somit eine Hilfestellung in der Etablierung interner Behandlungspfade, aber auch Anreize für die Entwicklung externer Kooperationen geben, damit die zunehmende Anzahl von Patienten mit z. T. komplexen ventrikulären Arrhythmien so wirksam wie möglich behandelt werden können. Ein interventionelles Vorgehen mit Ablation der vor allem für den elektrischen Sturm anzuschuldigenden monomorphen Kammertachykardien erscheint für alle Patienten aufgrund des hohen Rezidivrisikos geboten. Das optimale Timing der Katheterablation bleibt bisher unklar, allerdings erscheint eine frühe Intervention (wahrscheinlich im Rahmen des initialen Klinikaufenthalts) zweckmäßig. Bei Patienten mit fokal getriggerten polymorphen Kammertachykardien ist zwar die Katheterablation ebenfalls wirkungsvoll, allerdings fehlen hier aufgrund der geringen Patientenzahlen belastbare Daten zum mittel- oder langfristigen Outcome. Die Prävention eines weiteren elektrischen Sturms beinhaltet neben der katheterablativen Substratmodifikation auch die optimierte Herzinsuffizienztherapie, Prävention zukünftiger Myokardischämie-Ereignissen, eine durchdachte medikamentöse Therapie sowie eine optimale, individuell angepasste Programmierung implantierter Devices. Es bleiben viele Unklarheiten und Ungewissheiten über die Mechanismen des elektrischen Sturms, deren Klärung für die Etablierung angepasster Therapiestrategien notwendig erscheint.
Generell ist es bei Patienten mit elektrischem Sturm entscheidend, den Mechanismus der ventrikulären Arrhythmie zu verstehen, mögliche Trigger-Faktoren zu identifizieren, eine geeignete Therapie zur akuten aber auch zur mittelfristig anhaltenden Rhythmusstabilisierung einzuleiten und weitere Ereignisse eines elektrischen Sturms zu verhindern, um so die Prognose dieser Patienten zu verbessern.

Ich wünsche Ihnen auch im Namen aller Mitwirkenden viel Spaß und hoffentlich interessante Anregungen bei der Lektüre,

Thomas Deneke

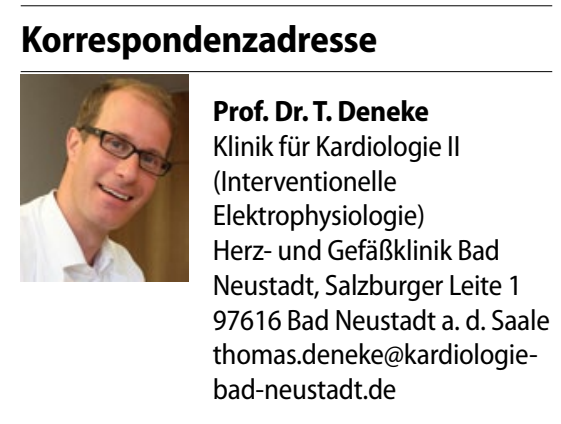

